Check for updates

Cite this: DOI: 10.1039/c8ra08744j

\title{
Novel fabrication of porous titanium by a resin- impregnated titanium substitution technique for bone reconstruction
}

\author{
Reiko Kobatake, Kazuya Doi, (DD * Takayasu Kubo, Yusuke Makihara, Yoshifumi Oki, \\ Miyuki Yokoi, Hanako Umehara and Kazuhiro Tsuga
}

\begin{abstract}
The purpose of this study was to develop a novel porous titanium material with superior mechanical strength and osteoconduction for bone reconstruction. Porous titanium samples were fabricated by titanium-slurry impregnate to prepare urethane forms with several porosities (high-porosity; 92\%, middle-porosity; $85 \%$ and low-porosity; 65\%). Porous HA (mean porosity; 75.3\%) was used as a control. To evaluate the characteristics of these materials, we performed porosity measurements, scanning electron microscopy (SEM), three-point bending testing, and cell proliferation assays. To evaluate the osteoconduction ability, porous titanium was placed into the femurs of rabbits and histological and histomorphometric evaluations were performed after 3 weeks. In SEM images, porous three-dimensional structures were observed in all samples. The bending strength significantly increased as porosity increased (Ti-65 > Ti-85 > porous HA > Ti-92, $P<0$.05; respectively). Ti-65, Ti-85, and porous HA showed good cell proliferation. Newly formed bone was observed in the central portion of Ti-65, Ti-85, and porous HA. Ti-92 was mainly detected in the bone marrow tissue. The bone formation areas of $\mathrm{Ti}-65, \mathrm{Ti}-85$, and porous $\mathrm{HA}$ were significantly higher than that of Ti-92 $(P<0.05)$. It was suggested that novel developed porous titanium composed of Ti-65 and Ti-85 showed superior mechanical strength and osteoconduction.
\end{abstract}

Received 22nd October 2018 Accepted 25th November 2018

DOI: $10.1039 / c 8 r a 08744 j$

rsc.li/rsc-advances biomechanical mismatch of elastic moduli, production of wear debris, and no supply of blood. ${ }^{12}$ Because it lacks osteoconductive ability, bone tissue does not ingrowth to the materials and bone formation at the defect area is insufficient.

Thus, a novel process for fabricating porous titanium of a suitable size with optimal porosity and an interconnected structure is required.

To overcome these limitations, we developed a novel porous titanium fabrication technique (the process is described below). The method can be used to produce a porous base resin substrate to prepare titanium structures.

This novel fabrication method can be used to develop a porous titanium with optimal porosity and interconnected porous structures as a bone reconstruction material.

The aim of the present study was to evaluate the characteristic structures and osteoconduction ability of porous titanium structures using a resin-impregnated titanium substitution technique.

\section{Experimental}

\section{Materials}

High-, middle-, and low-porosity urethane sponges were used as the base resin structures. These materials had porosities of $92 \%$, $85 \%$, and 65\%, respectively. Porous HA (NEOBONE®, Aimedic MMT Co. Ltd) was used as a control.
Department of Advanced Prosthodontics, Hiroshima University Graduate School of Biomedical and Health Sciences, 1-2-3, Kasumi, Minami-ku, Hiroshima 734-8553, Japan.E-mail: kazuya17@hiroshima-u.ac.jp; Fax: +81 82257 5679; Tel: +81 82257 5677 
1. Soaking the urethane into the titanium slurry

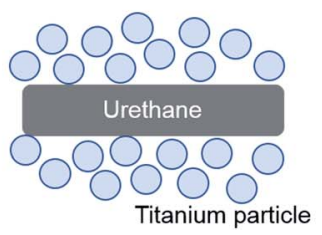

2. Impregnation the titanium into the urethane

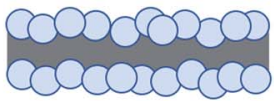

3. Elimination of the urethane at $600{ }^{\circ} \mathrm{C}$

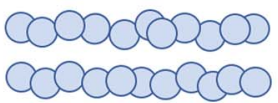

4. Sintering at $1300^{\circ} \mathrm{C}$

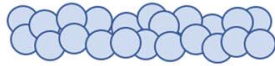

Fig. 1 Schematic representation of the resin-impregnated titanium substitution technique. The urethane base material was immersed in a titanium slurry to adsorb titanium particles on the surface. After the urethane was removed by $600{ }^{\circ} \mathrm{C}$ heat treatment, $1300{ }^{\circ} \mathrm{C}$ sintering was performed, whereby the titanium particles were sintered and bonded. Using this process, a porous titanium body was produced with a similar structure as the base urethane form.

\section{Fabrication of porous titanium}

A slurry was prepared by mixing commercial solid titanium powders (diameter: under $45 \mu \mathrm{m}$ and average $25 \mu \mathrm{m}$; TILOP45, OSAKA Titanium Technologies, Osaka, Japan) and a binder (acrylic resin and isopropyl alcohol). Urethane forms with several porosities were soaked into the slurry to impregnate the titanium. Titanium impregnated with urethane was heated at $600{ }^{\circ} \mathrm{C}$ to eliminate the urethane and binder. Carbonate present in the urethane base remained and titanium particles combined with each other. This material was sintered at $1300{ }^{\circ} \mathrm{C}$ in a vacuum (Fig. 1). In this process, porous titanium samples Ti-92, Ti-85, and Ti 65 were fabricated (Fig. 2a-c). These porous titanium samples were prepared in cylinders of three sizes: long type (diameter $6 \mathrm{~mm}$, height $60 \mathrm{~mm}$ ), short type (diameter $3 \mathrm{~mm}$, height $3 \mathrm{~mm}$ ), and disk type (diameter $13 \mathrm{~mm}$, height $2 \mathrm{~mm}$ ). Porous HA was used as a control (Fig. 2d).

\section{SEM observation}

The porous structures of each urethane form and porous titanium samples (Ti-92, Ti-85, and Ti-65) were evaluated at low magnification. Then surface structure of porous titanium and porous HA were also evaluated at high magnification. All samples were sputtered with Pt-Pd to make the surface conductive. Each sample was set on the sample stage with carbonate adhesive tape and examined by scanning electron microscopy (SEM, JOEL7200PLUS/LA, Nihon Denshi Oyo Co. Ltd., Tokyo, Japan).

\section{Measurement of porosity}

The total porosity of each short type samples were measured as the apparent volume calculated from the specimen dimensions and actual volume evaluated with a helium pycnometer (Accupyc II 1340, Micromeritics Instrument Corp., Norcross, GA) ( $n$ $=5) .^{13}$

\section{Measurement of mechanical strength}

Three-point bending tests were performed according to previous study. ${ }^{14}$ The long type porous Ti samples (Ti-92, Ti-85, and Ti-65) and porous HA were evaluated for mechanical strength. The distance between support points was set to $40 \mathrm{~mm}$ before the experiment. A load was continuously applied to the
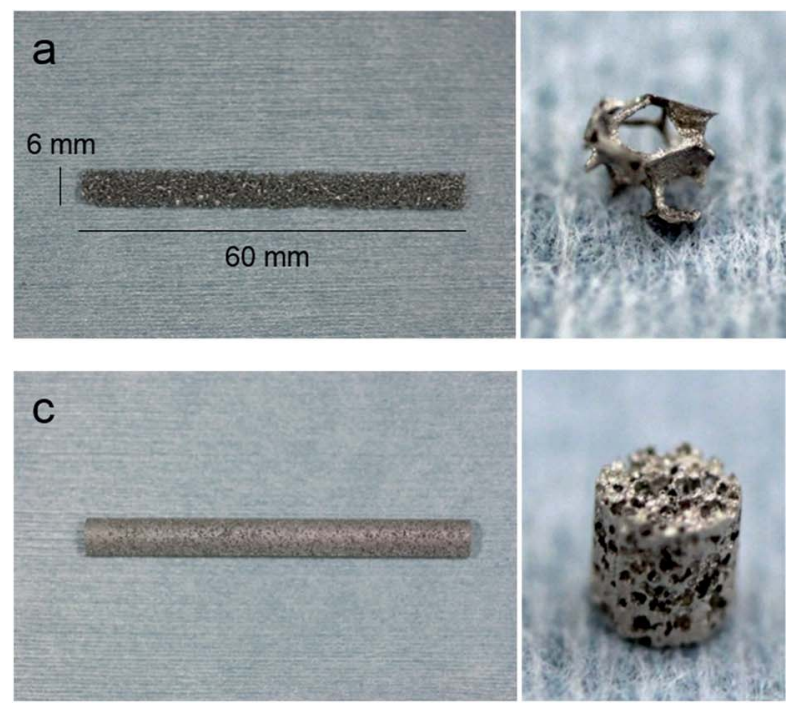

Fig. 2 Fabricated porous titanium and porous HA as control. (a) Ti-92: high-porosity titanium, (b) Ti-85: middle-porosity titanium, (c) Ti-65: lowporosity titanium, (d) porous HA. Fabricated porous titanium was cut into cylinders of two sizes: one was long (diameter $6 \mathrm{~mm}$, length $60 \mathrm{~mm}$ ) and the other was short (diameter $3 \mathrm{~mm}$, height $3 \mathrm{~mm}$ ).
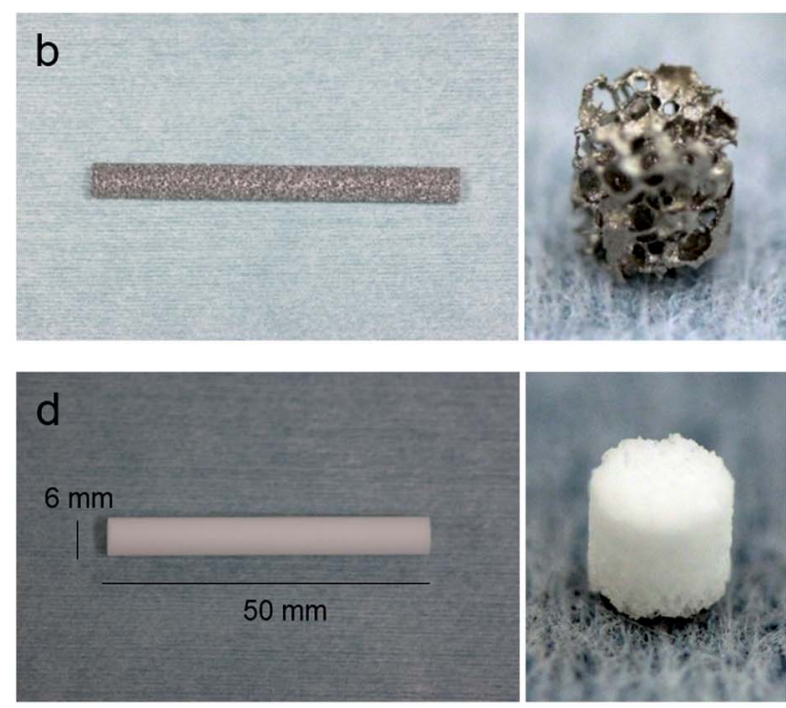


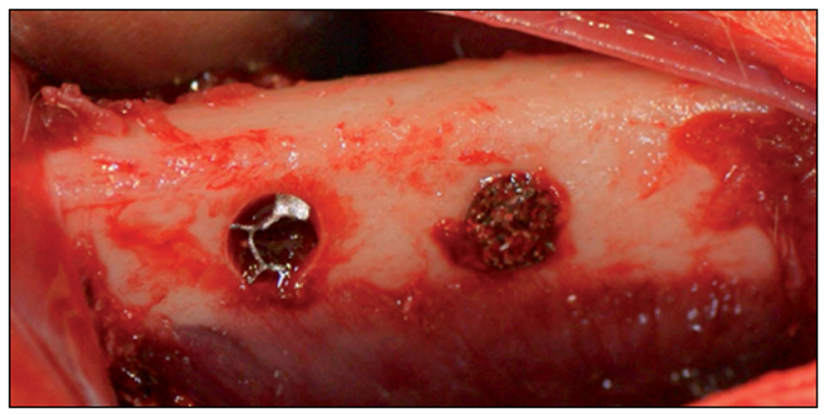

Fig. 3 Implantation. Two bone defects (diameter $3 \mathrm{~mm}$, length $3 \mathrm{~mm}$ ) on the rabbit femurs on both sides were prepared. Short type of each sample were randomly placed in the bone defects.

sample at a crosshead speed of $0.1 \mathrm{~mm} \mathrm{~min}^{-1}$ until fracture occurred (AUTO GRAPH AGS-X, Shimadzu). Then, bending strength was recorded as the mechanical strength (MPa).

\section{Cell proliferation assay}

The disk type porous Ti samples (Ti-92, Ti-85, and Ti-65) were used. Bone stromal cells were seeded onto the titanium disk of each porosity $(n=5)$ in 24-well culture plates with 200000 cells per well and incubated in normal culture medium (DMEM containing $10 \%$ fetal bovine serum and $10 \%$ penicillin-streptomycin). Three wells contained only medium for background subtraction. Cells were incubated for $4 \mathrm{~h}$ for initial attachment. The culture medium was changed every 3 days. After 4 h, 3 days, and 7 days of culture, cell proliferation was evaluated. After washing with phosphate-buffered saline, $1000 \mu \mathrm{L}$ medium and $100 \mu \mathrm{L}$ of cell counting kit 8 solution (Dojindo Laboratory,
Kumamoto, Japan) was added to each well. After incubation at $37{ }^{\circ} \mathrm{C}$ for $2 \mathrm{~h}$, we dispensed $100 \mu \mathrm{L}$ per well cell suspension of a 96-well plate, with 3 wells for each sample. The absorbance at $450 \mathrm{~nm}$ was measured with a microplate reader (MPR A4i, Tosoh Corporation, Tokyo, Japan). Absorbance at $450 \mathrm{~nm}$ was also measured at 3 days and 7 days of incubation.

\section{In vivo analysis}

All animal procedures were performed in accordance with the Guidelines for Care and Use of Laboratory Animals of Hiroshima University and Experiments were approved by the Animal Ethics Committee of Ministry of Education, Culture, Sports, Science and Technology-Japan. The study was approved by the Research Facilities Committee for Laboratory Animal Science, Hiroshima University School of Medicine (A11-5-5).

Four New Zealand white rabbits (17 W, male, 3.0-3.5 kg) were used. The surgical procedures were performed with the rabbits under general anaesthesia with sodium pentobarbital (10 $\mathrm{mg} \mathrm{kg}^{-1}$ ) and local infiltration anaesthesia with $2 \%$ lidocaine and $1: 80.000$ noradrenaline. Muscle and periosteal flaps were made on the left and right femurs, and two bone defects (diameter, $3 \mathrm{~mm}$; length, $3 \mathrm{~mm}$ ) on both sides were prepared. Each short type porous sample (Ti-92, Ti-85, and Ti-65) and porous HA as a control were randomly placed in the bone defects (Fig. 3). At 3 weeks after placement, the rabbits were anesthetized and perfused with 10\% neutral formalin through the aorta. Femurs were harvested and fixed in $10 \%$ neutral formalin for 1 week. The blocks were then dehydrated through a graded ethanol series and embedded in resin (Technovit 7200VLC, Kulzer, Wehrheim, Germany). The resin blocks were cut with a half cut of the sample and ground to approximately 70-80 $\mu \mathrm{m}$ thickness. The specimens were stained with toluidine
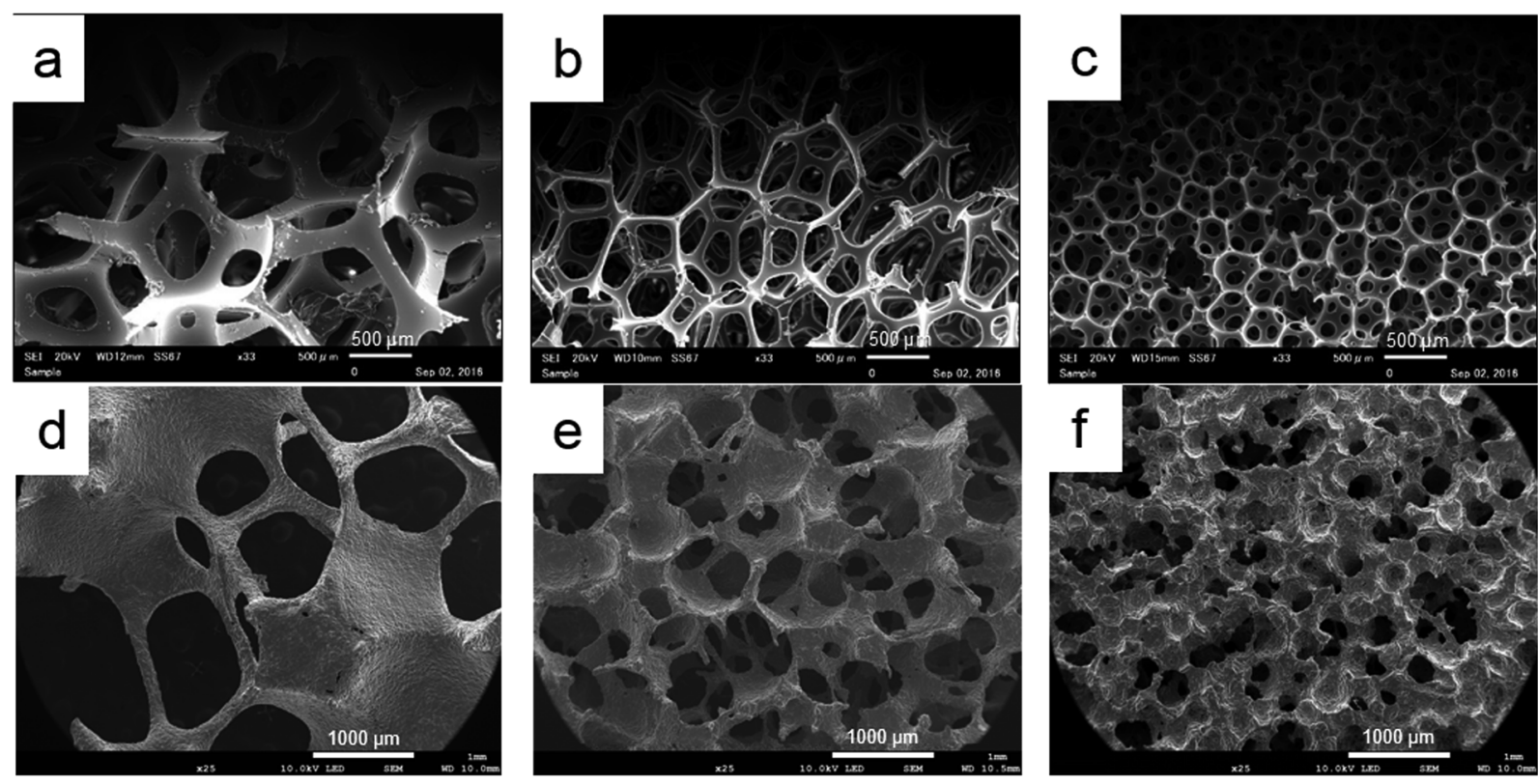

Fig. 4 Scanning electron microscopy observation of porous structure. (a) High-porosity urethane form, (b) middle-porosity urethane form, (c) low-porosity urethane form, (d) Ti-92, (e) Ti-85, (f) Ti-65. Fabricated porous titanium showed a cancellous structure similar to each base urethane form. However, in Ti-65, the microscopic interconnected porous structure was not reconstructed. 

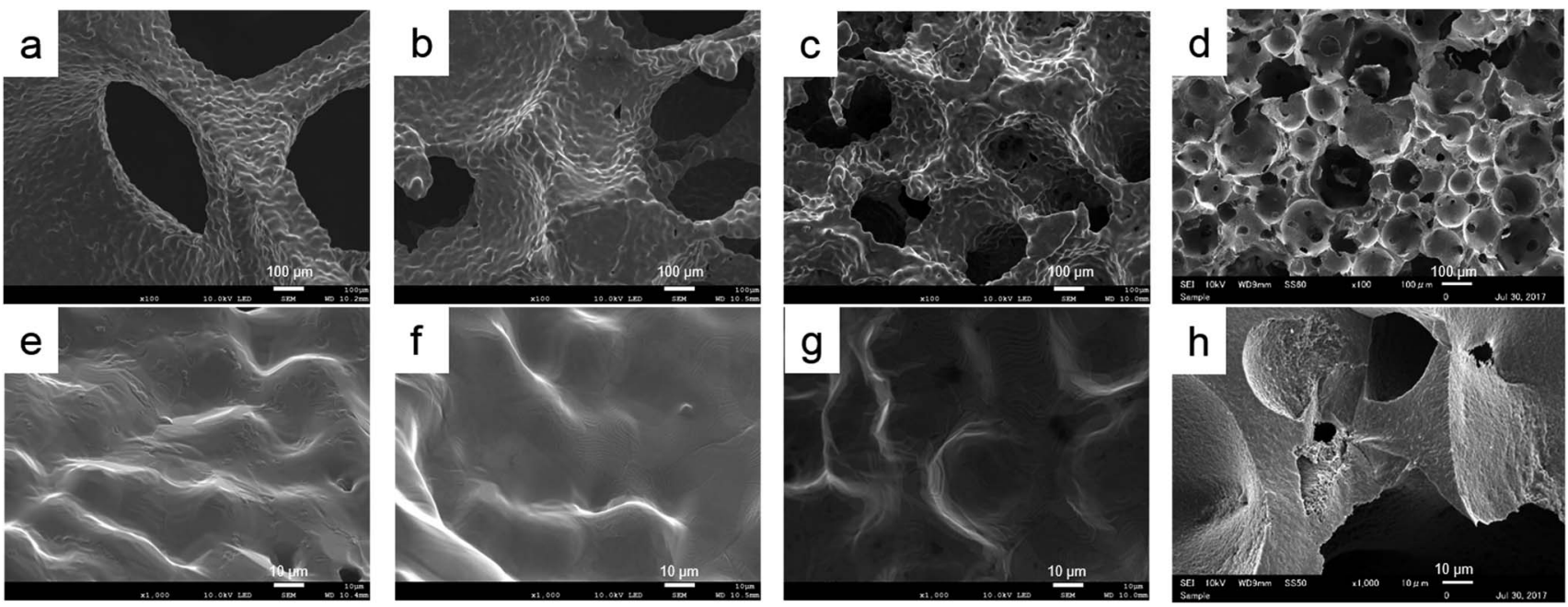

Fig. 5 Scanning electron microscopy observation of surface structure. (a and e) Ti-92, (b and f) Ti-85, (c and g) Ti-65, (d and h) porous HA. Fabricated porous titanium showed a smooth surface conditions each samples. Porous HA as control detected slight roughly surface rather than fabricated titanium.

blue and images of bone regeneration were digitized and histomorphometrically analysed using ImageJ software (National Institutes of Health, Bethesda, MD, USA). The bone formation ratio was calculated as the bone tissue in the pore area divided by the total tissue area in the pore. These bone formation ratios were statistically analysed at the $5 \%$ significance level using one-way analysis of variance and Tukey honestly significant difference multiple comparison assay $(n=5)$.

\section{Results}

\section{SEM observation}

The three-dimensional structures of fabricated porous titanium samples were similar to each base urethane form. However, in Ti-65, the microscopic interconnected porous structure was not reconstructed (Fig. 4). At high magnification, we observed a smooth surface that was convexo-concave in which titanium powders were sintered and bonded. For the outer structure, differences due to porosity were not observed. Porous HA was also had the interconnected porous structure and the surface was slight roughness (Fig. 5).

\section{Porosity}

Porosities of fabricated porous titanium samples tended to be slightly higher than those of the base urethane forms. Porous HA as a control showed $75.3 \pm 1.2 \%$ total porosity. Ti-92 and Ti85 of porous titanium showed higher porosities than porous HA (Table 1).

\section{Mechanical strength}

The mechanical strength was significantly increased as porosity decreased (Ti-65 > Ti-85 > porous HA > Ti-92). Ti-65 and Ti-85 were significantly higher than that of porous HA (Table 2).

\section{Cell proliferation assay}

At 3 days, cell proliferation on Ti-85 was significantly higher than that on Ti-92. At 7 days, Ti-85 and Ti-65 were significantly higher than on Ti-92, and the difference between Ti-85 and Ti65 was not significant (Fig. 6).

\section{In vivo analysis}

In the histological analysis results of porous titanium and porous HA at 3 weeks after placement, the yellow dot square indicates the placed porous titanium or porous HA area. Newly formed bone in the pores was observed in the marginal portion of all groups. The central portion of Ti-92 was mainly occupied by marrow tissue. In contrast, in Ti-85 and Ti-65, high levels of bone formation were observed even in the centre of the pores as observed in the control (Fig. 7).

Table 1 Porosity (\%)

\begin{tabular}{llc}
\hline & Urethane & Porous Ti/HA \\
\hline Ti-92 & 92 & $93.9 \pm 1.5$ \\
Ti-85 & 85 & $85.3 \pm 2.6$ \\
Ti-65 & 65 & $69.7 \pm 1.9$ \\
Porous HA & & $75.3 \pm 1.6$
\end{tabular}

Table 2 Mechanical strength

Bending strength (MPa)

Ti-92

Ti-85

Ti-65

Porous HA

${ }^{a}$ Ti-85 compared to Ti-92 and porous HA $(P<0.05) .{ }^{b}$ Ti-65 compared to another groups $(P<0.05) .{ }^{c}$ Porous HA compared to Ti-92 $(P<0.05)$. 


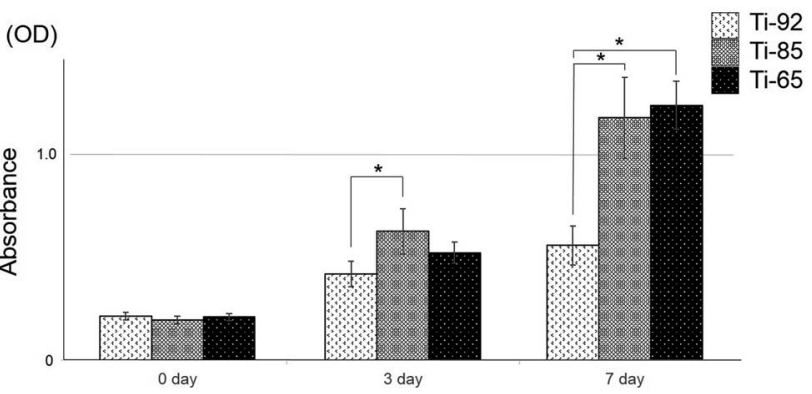

Fig. 6 Cell proliferation. In 0 day, there are no significant difference in three groups. In 3 day, Ti-85 was significantly higher than Ti-92 $(P<$ 0.05). In 7 days, Ti- 85 and Ti-65 were significantly higher than Ti-92 $(P$ $<0.05)$.

\section{Bone formation ratio}

The bone formation ratios of Ti-85, Ti-65, and control groups were significantly higher than that of Ti-92. Additionally, there were no significant differences between the Ti-85, Ti-65, and control groups (Fig. 8).

\section{Discussion}

Bone reconstruction materials should have good biocompatibility, show sufficient mechanical strength, recover bone form before loss (obtain the optional form and structure), and exhibit good osteoconductive ability. ${ }^{15}$ Titanium has good biocompatibility and sufficient mechanical strength, and the porous structure is advantageous for natural bone growth into pores. ${ }^{16}$

Therefore, numerous studies have attempted to develop methods for fabricating porous titanium structures. The most widely used and traditional process for producing porous titanium is sintering of titanium powders. ${ }^{\mathbf{1 7 - 2 0}}$ The porous structure is prepared with a gap space integrated into each titanium powder by sintering. Using this method, it is difficult to ensure uniform porosity and impart high porosity. ${ }^{21}$ Titanium spray or laser melting methods are used to fabricate porous titanium structures. ${ }^{22}$ However, it is difficult to set the interconnected structures. Additionally, in both methods, it is difficult to fabricate porous titanium with a large size.

We fabricated porous titanium with a structure similar to that of the base urethane form with arbitrary porosity and a three-dimensional interconnected porous structure. In SEM observation, the pores were interconnected in all samples. The pore size was approximately $200-300 \mu \mathrm{m}$ in Ti-65 and Ti-85. In Ti-92, the pore size was more than $600 \mu \mathrm{m}$. Some reports showed that pore sizes of $200-500 \mu \mathrm{m}$ were optimal for the colonization of osteoblasts, fibroblasts, or vascular ingrowth of new bone. ${ }^{12,23}$ The pore sizes of Ti-85 and Ti-65 were in this range. However, some pores in Ti-65 were partly isolated. This is because the titanium slurry flowed and adhered into the interconnected pores of the base material, followed by sintering and bonding.

The porosities of the base urethane form were $92 \%, 85 \%$, and $65 \%$ for high, middle, and low porosity. We predicted that high porosity could be achieved by increasing the urethane porosity condition; however, if the porosity was further increased to $92 \%$, the porous structure was not maintained. In contrast, for a lower porosity, the pores would be crushed, and the interconnected porous structure would not be maintained. Thus, we set the maximum and minimum within the technical range, and the middle porosity was set according to the pore size of $300 \mu \mathrm{m}$, which is suitable for bone formation.

It was possible to produce a porous structure with porosity similar to the base urethane; however, higher porosity was observed in titanium compared to in the base urethane form. This is because during the fabrication process, titanium particles on the surface of the base skeleton were sintered and bonded. It is considered that the skeleton became slightly thinner than the original urethane. Since the titanium powder with a small particle diameter is used, the porosity of the framework itself becomes small.

In early stages of implantation, excellent mechanical support is required for bone reconstruction. ${ }^{24}$ Fragile bone reconstruction materials fail at an early stage. Additionally, fragile materials cannot be used for large bone defects or load-bearing situations. In this study, the mechanical strength was evaluated in a three-point bending test and the strength at the breaking
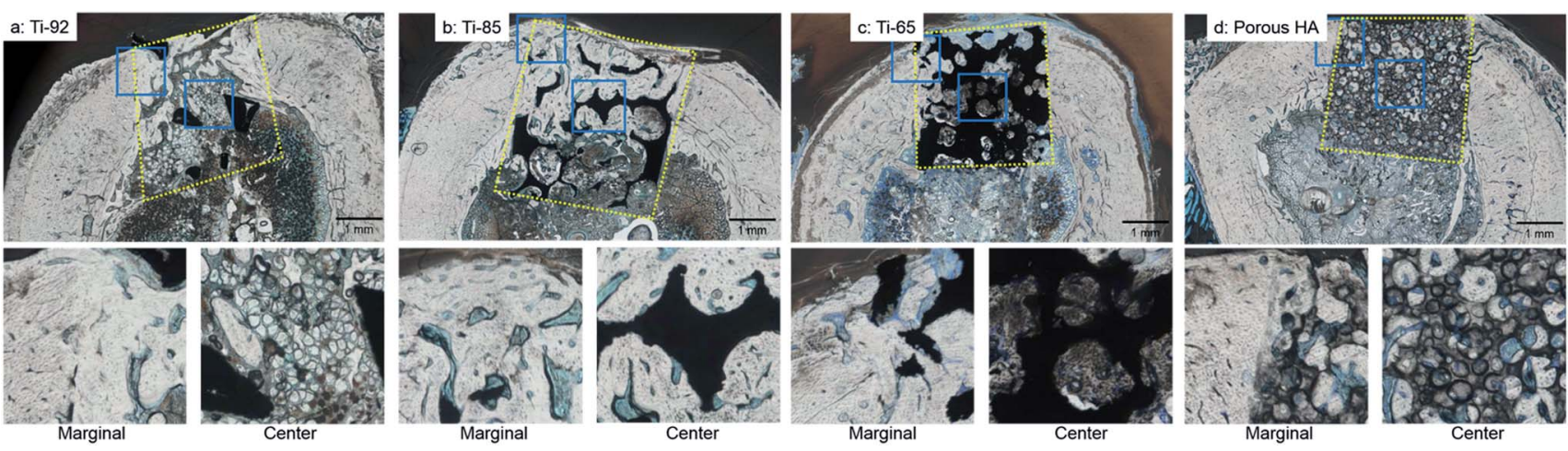

Fig. 7 Histological observation. In the histological analysis results of porous titanium and porous HA at 3 weeks after placement, the yellow dot square indicates the placed sample area and titanium shown in black. (a) (Marginal) Newly formed bone in the pores from parent bone was observed. (Central) It was mainly occupied by marrow tissue. (b-d) (Marginal) Newly formed bone in the pores from parent bone was observed. (Central) High levels of bone formation were observed even in the centre of the pores and osteocytes were detected. 


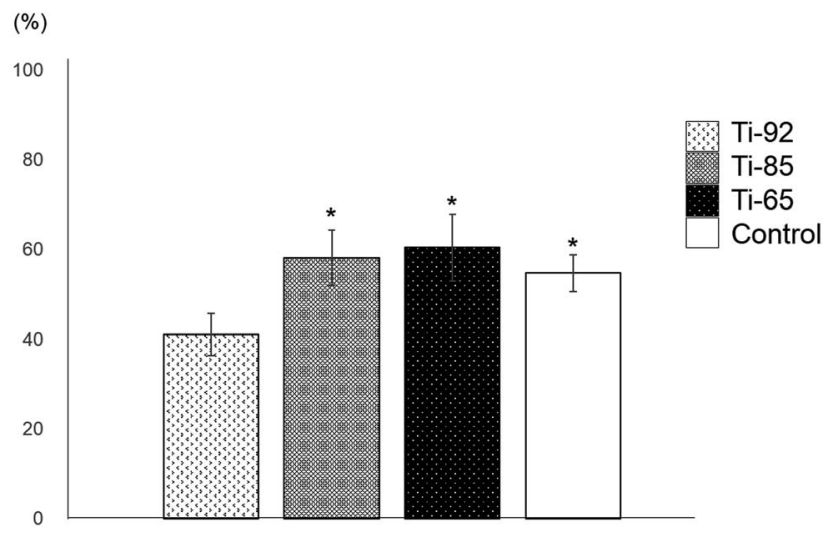

Fig. 8 Bone formation ratio. Ti-85, Ti-65, and porous HA were significantly higher than Ti-92. Additionally, there were no significant differences between Ti-85, Ti-65, and porous HA $(P<0.05)$.

point was measured. The three-point bending test is widely used to evaluate mechanical strength.

As the porosity of porous Ti decreases, mechanical strength increases. For application to bone defects at load-bearing sites, the mechanical strength of a biomaterial plays a key role in bone reconstruction. The mechanical strength of Ti-92 was lower than that of control porous HA and is insufficient strength for bone reconstruction. ${ }^{25}$

The mechanical strength of porous titanium was negatively related to porosity. Particularly, the Ti-85 showed high strength despite its higher porosity compared to porous HA as a control. However, the standard deviation of mechanical strength was slightly high in Ti-65 titanium. This indicates that thin and thick areas existed in the skeleton of the titanium porous body. When the force of three-point bending was applied to the thick part of the skeleton, a large value was observed.

Cell proliferation assay was higher on Ti-65 and Ti-85 than on Ti-92. This increase in cell proliferation may be attributed to the optimal three-dimensional porous structure. As described above, suitable pore sizes for cell attachment and proliferation are $200-500 \mu \mathrm{m} .{ }^{25}$ Additionally, surface area is related to cell attachment. Thus, cell counts were increased in Ti-65 and Ti-82. These features are also related to bone formation ability. In later stages, bone formation on the materials is important. ${ }^{24}$ The created defect in this study was a critical size, ${ }^{26}$ and without application of a bone reconstruction material, the defect would not heal and fill with new bone in the central portion. We performed histological evaluation at the marginal area and central area. Under all conditions in the marginal area, new bone formation and bone conduction continuing from existing bone was observed. In the central portion, for Ti-65 and Ti-85, bone formation was observed even the centre of the pores and in the control, while for Ti-92, little bone tissue was observed. This material showed insufficient bone formation compared to other groups and was occupied by marrow tissue because the scaffold was insufficient for the defect space. This agrees with the results of the cell proliferation assay. We used porous HA as a control because of its three-dimensional interconnected structure and biocompatibility. Our previous studies demonstrated good osteoconduction and scaffold abilities of this biomaterial. ${ }^{2}$ In vivo, porous titanium of Ti-65 and Ti-85 showed good osteoconduction relative to porous HA.

We successfully fabricated porous titanium with optimal porosity, an interconnected pore structure, and good mechanical strength for use as bone reconstruction materials. A bone reconstruction material must recover the bone form before loss. Using the methods, the urethane form can be used for titanium as a base model. Because urethane can easily form any structure, it is possible to fabricate large and specific shapes. Porous titanium with $65-85 \%$ porosity showed good mechanical strength and osteoconduction and is suitable as a bone reconstruction material.

\section{Conclusions}

We determined the properties and osteoconduction ability of developed porous titanium structures using novel fabrication method. Ti-65 and Ti-85 had appropriate properties and osteoconduction ability. Newly fabricated porous titanium might be useful as a bone reconstruction material even under load-bearing conditions.

\section{Conflicts of interest}

There are no conflicts to declare.

\section{Acknowledgements}

This study was supported by a Scientific Research Grant (No. 18K09683) from the Japan Society for the Promotion of Science.

\section{References}

1 N. Tamai, A. Myoui, T. Tomita, T. Nakase, J. Tanaka, T. Ochi and H. Yoshikawa, J. Biomed. Mater. Res., 2002, 59, 110-117.

2 T. Kubo, K. Doi, K. Hayashi, K. Morita, A. Matsuura, E. R. Teixeira and Y. Akagawa, J. Prosthodont. Res., 2011, 55, 104-109.

3 H. Shigeishi, M. Takechi, M. Nishimura, M. Takamoto, M. Minami, K. Ohta and N. Kamata, Dent. Mater. J., 2012, 31, 54-60.

4 K. Doi, T. Kubo, R. Takeshita, S. Kajihara, S. Kato, Y. Kawazoe, T. Shiba and Y. Akagawa, Dent. Mater. J., 2014, 33, 179-186.

5 E. Martz, V. Goel, M. Pope and J. Park, J. Biomed. Mater. Res., 1997, 38, 267-288.

6 K. Rezwan, Q. Z. Chen, J. J. Blaker and A. R. Boccaccini, Biomaterials, 2006, 27, 3413-3431.

7 G. Ryan, A. Pandit and D. P. Apatsidis, Biomaterials, 2006, 27, 2651-2670.

8 B. Yin, B. Xue, Z. Wu, J. Ma and K. Wang, Am. J. Transl. Res., 2018, 15, 474-482.

9 D. Kuroda, M. Niinomi, M. Morinaga, Y. Kato and T. Yashiro, Mater. Sci. Eng., A, 1998, 243-244.

10 W. F. Ho, C. P. Ju and C. H. C. Lin, Biomaterials, 1999, 20, 2115. 
11 D. M. Brunette, P. Tengvall, M. Textor and P. Thomsen, Titanium in Medicine, Springer-Verlag, Berlin, 2001.

12 C. E. Wen, Y. Yamada, K. Shimojima, Y. Chino, H. Hosokawa and M. Mabuchi, J. Mater. Res., 2002, 45, 1147-1153.

13 F. J. Semel and D. A. Lados, Powder Metall., 2009, 52, 282290.

14 K. Morita, K. Doi, H. Oue, S. Kajihara, K. Hayashi and Y. Akagawa, Br. J. Oral Maxillofac. Surg., 2013, 51, 550-554.

15 V. Karageorgiou and D. Kaplan, Biomaterials, 2005, 26, 54745491.

16 H. Yoshikawa, N. Tamai, T. Murase and A. Myoui, J. R. Soc., Interface, 2009, 6, 341-348.

17 I. H. Oh, N. Nomura and S. Hanada, Mater. Trans., 2002, 43, 443-446.

18 I. H. Oh, N. Nomura, N. Masahashi and S. Hanada, Scr. Mater., 2003, 49, 1197-1202.
19 D. C. Dunand, Adv. Eng. Mater., 2004, 6, 369-376.

20 M. Guden, E. Celik, A. Hizal, M. Altindis and S. Cetiner, J. Biomed. Mater. Res., Part B, 2008, 85, 547-555.

21 B. Arifvianto and J. Zhou, Materials, 2014, 7, 3588-3622.

22 S. Fujibayashi, M. Neo, H. M. Kim, T. Kokubo and T. Nakamura, Biomaterials, 2004, 25, 443-450.

23 W.Frieb, J.Warner, F.Schuth, K. S. W.Sing and J.Weitkamp, Handbook of Porous Solids, Wiley-VCH, Weinheim, 2002.

24 J. Yang, H. J. Chen, X. D. Zhu, S. Vaidya, Z. Xiang, Y. J. Fan and X. D. Zhang, J. Mater. Sci.: Mater. Med., 2014, 25, 1747-1756.

25 M. Zhang, G. L. Wang, H. F. Zhang, X. D. Hu, X. Y. Shi, S. Li and W. Lin, Artif. Organs, 2014, 38, 493-502.

26 T. J. Levingstone, E. Thompson, A. Matsiko, A. Schepens, J. P. Gleeson and F. J. O'Brien, Acta Biomater., 2016, 32, 149-160. 